\title{
Mathematical modelling of batch sedimentation subject to slow aggregate densification
}

\author{
Yi Zhang ${ }^{\mathrm{a}}$, Paul Grassia ${ }^{\mathrm{a}, *}$, Alastair Martin ${ }^{\mathrm{b}}$, Shane P. Usher ${ }^{\mathrm{c}}$, \\ Peter J. Scales ${ }^{\mathrm{c}}$ \\ ${ }^{a}$ CEAS, The Mill, University of Manchester, Oxford Road, Manchester M13 9PL, UK \\ ${ }^{b}$ Department of Engineering, Lancaster University, Bailrigg, Lancaster LA1 $4 Y R$ \\ ${ }^{c}$ Department of Chemical and Biomolecular Engineering, University of Melbourne, VIC \\ 3010, Australia
}

\begin{abstract}
This paper considers an initially networked suspension in a batch settler subjected to very slow aggregate densification. The so called pseudo-steady state aggregate densification theory developed by van Deventer (2012) has been extended to the case of initially networked suspensions. The solids behaviour and the evolutions of the suspension height and the consolidated bed height in the batch settler have been predicted using the extended pseudo-steady state theory. Different formulae for the weight-bearing strength of the consolidated bed (so called weak gel and strong gel formulae, which differ near the top of the bed) are considered. The suspension height approaches the consolidated bed height far more quickly when using the weak gel formula than when using the strong gel one. This paper also investigates how the initial feed solids volume fraction and the initial suspension height affect the evolutions of the heights of the suspension and the consolidated bed, as well as the determinations of the solids volume fractions obtained at the bottom
\end{abstract}

\footnotetext{
${ }^{*}$ Corresponding author

Email address: paul.grassia@manchester.ac.uk (Paul Grassia)
} 
of the batch settler. When the initial feed solids fraction is sufficiently large and/or the initial suspension is sufficiently tall, the densification process has little effect on the solids fraction observed at the bottom of the settler.

Keywords: Gels; Rheology; Suspension; Mathematical modelling;

Compressive yield stress; Sedimentation

\section{Introduction}

Marked revision: Section 1 has been entirely rewritten and restructured. It has been shortened significantly compared to the original submission.

Batch settling under gravity is an elementary solid-liquid separation process, concentrating typically denser solids relative to surrounding less dense liquid (Kynch, 1952). It is important not only in its own right, but also as a technique for extracting suspension rheological properties (Kynch, 1952; Lester et al., 2005; Diehl, 2007; Stickland et al., 2008; Grassia et al., 2008, 2011), these properties subsequently being used for designing other solidliquid separation devices, including continuous thickeners (Landman et al., 1988; Bürger and Concha, 1998; Bürger et al., 1999; Martin, 2004; Usher and Scales, 2005), pressure filters (Landman et al., 1995; Landman and White, 1997), or centrifuges (Berres et al., 2005; Stickland et al., 2006).

Often in batch settling and related dewatering processes, one is dealing with flocculated suspensions (Landman and White, 1994), flocculants being added to the system to bind solids together into aggregates. Such aggregates generally contain a significant amount of liquid in addition to solids. However if the aggregate is sheared (e.g. under the action of a rake (Farrow et al., 2000) and/or by being buffeted by neighbouring aggregates (Spehar et al., 2014)), 
it can undergo a process of densification, i.e. the solids in the aggregate bind together more tightly by expelling liquid (Farrow et al., 2000). Shear can therefore have a very significant effect on suspension dewatering (Gladman, 2004; Gladman et al., 2005, 2010).

The end state of the batch settling process (Howells et al., 1990) typically involves a consolidated bed of solid-liquid aggregates networked together, with clear liquor (free of solids) situated higher up. The more tightly the aggregates bind together as a result of densification, the higher the overall solids fraction in the networked bed (overall solids fraction being the product of the solids fraction in the aggregates and the fraction of space filled by them), and hence the better the solid-liquid separation that is achieved.

In addition to affecting the end state of the solid-liquid separation process, shear-induced aggregate densification also affects the rate of achieving that separation. Given that a densified aggregate has the same buoyancy force relative to surrounding liquid as it had prior to densification, but experiences less viscous drag, it therefore settles faster (Usher et al., 2009).

There have been a number of recent studies in the literature attempting a mathematical description of aggregate densification (Usher et al., 2009; van Deventer et al., 2011), and its effects on suspension dewatering processes (Zhang et al., 2013a,b; Grassia et al., 2014). These introduced a so called densification rate parameter (van Deventer et al., 2011; Zhang et al., 2013a; Grassia et al., 2014), the reciprocal of this rate parameter giving a characteristic time for aggregates to densify. There are, as a result, three relevant time scales applicable to solid-liquid separation in a sheared batch settler: a characteristic sedimentation time (i.e. the suspension height di- 
vided by the suspension settling speed), the residence time of the suspension in the batch settler, and the aforementioned densification time.

There is a physically meaningful limit corresponding to 'slow shear' (van Deventer, 2012), for which the characteristic sedimentation time is much shorter than either the residence time or the densification time ${ }^{1}$. In this 'slow shear' limit, the suspension dewaters to the equilibrium state of an undensified suspension, but then continues to undergo further dewatering through a sequence of 'pseudo-steady states' as densification proceeds, binding solids in aggregates together increasingly tightly, allowing more and more liquid to be expelled.

The pseudo-steady state work of van Deventer (2012), even though it considered the final state of the suspension to be a networked bed (this networked bed being sufficiently strong to bear the suspension's weight), assumed the initial solids fraction to be sufficiently low that aggregates were isolated from one another (i.e. not networked together).

Another situation can however be contemplated: even in the initial state, the solids instead of being isolated from each other, could be slightly overlapped. Hence they form a network, albeit a weak one. Situations in which such a state could arise include laboratory characterisation of gelled suspensions and also consolidation in tailings impoundments. Whilst the weak network can bear some weight, it cannot bear the full weight of the suspension that is present, so some consolidation (typically towards the bottom of

\footnotetext{
${ }^{1}$ Such a limit could not normally be contemplated in another device such as a continuous thickener (Usher and Scales, 2005), because for a thickener, characteristic sedimentation time and the residence time in the device tend to be comparable (by design).
} 
the suspension) definitely must occur. However some solids (towards the top) remain at the initial solids fraction (Buscall and White, 1987).

Adding shear (and thereby aggregrate densification) to the case of an initially networked suspension can now lead to some quite complex behaviours. Since individual aggregates bind together more tightly as they densify, the degree of overlap with neighbours can reduce over time for a given overall solids fraction. Thus as a result of the action of shear, the weight bearing strength of the material decreases (Channell and Zukoski, 1997). Moreover the amount of suspension that is retained at the initial solids fraction without having yet consolidated gradually becomes less, and (depending on the system under consideration) might or might not disappear altogether. The aim here to is explore quantitatively the range of behaviours that can arise for an initially networked suspension subject to aggregate densification in the pseudo-steady state limit.

The remainder of this work is laid out as follows. Section 2 describes the pseudo-steady state batch settling model for networked suspensions incorporating aggregate densification and sets up a number of case studies. Results of these case studies are presented in Section 3. Conclusions are given in Section 4.

\section{Model and governing equations}

Marked revision: Section 2 has been completely rewritten and restructured, and has been shortened significantly compared to the original submission. All assumptions and governing equations are now contained within this section, instead of being dispersed over the whole text. 
In this section a description is given of the pseudo-steady state batch settling model including the effects of aggregate densification. The discussion is organised as follows. Section 2.1 explains how to determine the pseudosteady state given the initial suspension solids fraction, the initial height of suspension, and the suspension's rheological material properties. Section 2.2 then details the rheological material properties that must be considered, with two slightly different possible rheologies being discussed. After that Section 2.3 explains how aggregate sizes evolve due to densification. The effects of densification upon the suspension rheological properties are described in Section 2.4. For any given initial solids fractions, the particular rheologies that are assumed, coupled to the initial suspension height turn out to affect the solids fraction at the bottom of the suspension: this is explained in Section 2.5. Following this, Section 2.6 defines a number of case studies that will be analysed later on in the results section of the paper. For increased generality, case study results will be presented in dimensionless form using a set of conversions outlined in Section 2.7.

More information about the models and underlying assumptions can be found in Usher et al. (2009); van Deventer et al. (2011); Zhang et al. (2013a,b); Grassia et al. (2014).

\subsection{Pseudo-steady state suspension}

An initially uniform networked suspension (at an initial 'feed' solids volume fraction $\phi_{f}$ ) that settles to (pseudo-)steady state, generally consists of a consolidated bed with solids fraction $\phi>\phi_{f}$ below, a clear liquor zone (with no solids) above, and a column of unconsolidated material (still with $\phi=\phi_{f}$ ) 
retained between the two: see Figure 1.

The (pseudo-)steady state network stress in the suspension $P_{s}$ satisfies

$$
\frac{\partial P_{s}}{\partial z}+\Delta \rho g \phi=0
$$

where $\Delta \rho$ is the density difference between solid and liquid (chosen to be $2200 \mathrm{~kg} \mathrm{~m}^{-3}$ here), $g$ is gravity acceleration $\left(9.8 \mathrm{~m} \mathrm{~s}^{-2}\right)$ and $z$ is a coordinate measured upward from the bottom of the suspension. By definition $P_{s}$ vanishes at the interface between the suspension and the clear liquor zone, but increases moving downwards into the suspension.

The networked suspension is a gel that can resist compression up to a certain network stress that is called the compressive yield stress and that is denoted $P_{y}$. This $P_{y}$ is an increasing function of solids fraction $\phi$ : the higher the solids fraction, the greater the suspension's weight bearing strength.

In the unconsolidated column at $\phi=\phi_{f}$, one has $P_{s}<P_{y}\left(\phi_{f}\right)$. In the consolidated bed with $\phi>\phi_{f}$ however, the bed yields so as to maintain $P_{s}=P_{y}(\phi)$, with both $P_{s}$ and $\phi$ varying with $z$. It follows

$$
\frac{\partial P_{y}}{\partial \phi} \frac{\partial \phi}{\partial z}+\Delta \rho g \phi=0
$$

This equation gives a way to determine the profile of solids fraction $\phi$ vs $z$ in the bed provided the suspension material property $P_{y}$ vs $\phi$ is known.

Equation (2) applies from the bottom of the suspension $z=0$ up to some (a priori unknown) bed height $z=z_{c}$. Uniform solids fraction $\phi_{f}$ then applies 
up to the suspension height $z=H$. Since $P_{s}$ attains the value $P_{y}$ at $z=z_{c}$

$$
H=z_{c}+\frac{P_{y}\left(\phi_{f}\right)}{\Delta \rho g \phi_{f}}
$$

Provided the initial suspension height $H_{i}$ is known, the value of $z_{c}$ is then defined by a mass balance

$$
\int_{0}^{z_{c}} \phi \mathrm{d} z+\left(H-z_{c}\right) \phi_{f}=\phi_{f} H_{i}
$$

Obtaining $z_{c}$ however is more straightforward, if one integrates (1) from $z=0$ to $z=H$ and substitutes from (4) to obtain

$$
P_{y}\left(\phi_{b e}\right)=\Delta \rho g \phi_{f} H_{i} \equiv \Delta \rho g V_{s}
$$

where $\phi_{b e}$ is the solids fraction on the bottom boundary and $V_{s} \equiv \phi_{f} H_{i}$ is the total volume of solids in the suspension (per unit cross section). Thus $\phi_{b e}$ can be obtained if the function $P_{y}$ vs $\phi$ is known, and finally from (2)

$$
z_{c}=\int_{\phi_{f}}^{\phi_{b e}} \frac{1}{\Delta \rho g \phi} \frac{\partial P_{y}}{\partial \phi} \mathrm{d} \phi
$$

\subsection{Compressive yield stress}

The compressive yield stress $P_{y}$ is a suspension material property. Below a particular solids fraction (the suspension gel point, denoted $\phi_{g}$ ) the compressive yield stress is identically zero (Landman and White, 1994): the suspension has not yet formed a network and cannot bear weight. For $\phi>\phi_{g}$, the compressive yield stress is non-zero, and experimental techniques are 
available for measuring it for any given suspension (Green et al., 1996, 1998; de Kretser et al., 2001; Usher et al., 2001).

Here particular functional forms for $P_{y}$ (containing a number of fitting parameters) are used which have been employed in previous studies (Usher et al., 2009; Zhang et al., 2013a,b). The parameters have been selected to be typical for suspensions present in large-scale industrial thickeners (Usher et al., 2009).

One previous study (Zhang et al., 2013b) however showed that dewatering behaviour can be remarkably sensitive to fine details of how $P_{y}$ varies in the neighbourhood of $\phi_{g}$. That study therefore distinguished two cases: a 'weak gel' (compressive yield stress denoted $P_{w y}$ ) for which $\partial P_{w y} / \partial \phi$ grows gradually away from zero as $\phi$ exceeds $\phi_{g}$, and a 'strong gel' (compressive yield stress $\left.P_{s y}\right)$ such that $\partial P_{s y} / \partial \phi$ jumps to a finite value at $\phi=\phi_{g}$.

To begin with, the compressive yield stress is considered in the absence of aggregate densification. In this undensified state, the weak and strong gel formulae are denoted by $P_{w y, 0}$ and $P_{s y, 0}$, and the gel point is denoted by $\phi_{g, 0}$. It is assumed

$$
P_{w y, 0}=C_{0}\left(\frac{\left(\phi-\phi_{g, 0}\right)}{\left(b+\phi-\phi_{g, 0}\right)\left(\phi_{c p}-\phi\right)}\right)^{k_{0}}
$$

with parameter values $C_{0}=3.1866 \mathrm{~Pa}, b=0.002$, and $k_{0}=11$ (Usher et al., 2009; Zhang et al., 2013b). The undensified gel point, $\phi_{g, 0}$ is chosen to be 0.1 , whilst $\phi_{c p}$ (a solids fraction which can never be exceeded because $P_{w y, 0} \rightarrow \infty$ there) is chosen as 0.8 (Usher et al., 2009; Zhang et al., 2013b). Meanwhile

$$
P_{s y, 0}=\frac{c_{0}\left(\phi-\phi_{g, 0}\right)}{\left(b_{1}+\phi-\phi_{g, 0}\right)\left(\phi_{c p}-\phi\right)^{K_{o}}}
$$


where $c_{0}=3.7914 \mathrm{~Pa}, b_{1}=0.0363$, and $K_{0}=10.8302$ (Zhang et al., 2013a) and $\phi_{g, 0}$ and $\phi_{c p}$ are as above.

Equations (7)-(8) are specific to undensified systems. To describe how the compressive yield stress varies due to aggregate densification, it is necessary first to model the densification process itself, as per the next section.

\subsection{Modelling aggregate densification}

It is assumed that aggregates have a solids fraction $\phi_{\text {agg }}$. Normally $\phi$ differs from $\phi_{a g g}$. If $\phi<\phi_{a g g}$, then the aggregates do not fill space but instead there are gaps between them: $\phi$ is then the product between $\phi_{\text {agg }}$ and the fraction of space that the aggregates occupy. On the other hand if $\phi>\phi_{\text {agg }}$, the aggregates fill space, and are completely interlinked and interpenetrating, to the extent that they no longer appear as discrete objects.

The role of densification is to change the aggregate diameter. Suppose $d_{a g g}$ is the diameter after densification, and $d_{a g g, 0}$ is the diameter before densification, with $D_{a g g} \leq 1$ being the diameter ratio $d_{a g g} / d_{a g g, 0}$. It has been proposed van Deventer et al. (2011); Zhang et al. (2013a) that $D_{\text {agg }}$ can be represented by a function of time

$$
D_{\text {agg }}=D_{\text {agg, } \infty}+\left(1-D_{a g g, \infty}\right) \exp (-A t) .
$$

Here $D_{a g g, \infty}$ is the ratio between the diameter of a 'fully densified' aggregate ${ }^{2}$ and an undensified one. Meanwhile $t$ denotes time and $A$ is an aggregate

\footnotetext{
${ }^{2}$ Note that 'fully densified' here does not mean that the solids in the aggregate are close packed together, but instead merely that the shear has ceased to drive further reductions in the aggregate diameter.
} 
densification rate parameter.

Previous work (Usher et al., 2009; van Deventer et al., 2011; Zhang et al., 2013a,b; Grassia et al., 2014) has employed the value $D_{a g g, \infty}=0.9$ which is also adopted here. Since the interest here is ultimately in a pseudo-steady state model here, the value of $A$ does not strictly speaking need to be specified: changing the value of $A$ merely changes the rate at which the system evolves through a given sequence of pseudo-steady states. For the sake of definiteness, it is possible to suppose $A \sim 0.0001 \mathrm{~s}^{-1}$ which is claimed to fall within the pseudo-steady state regime (van Deventer, 2012).

Changes in $D_{a g g}$ with time, imply changes in $\phi_{\text {agg }}$. Since the mass of solids is conserved in each aggregate during densification, it follows that $\phi_{\text {agg }}=\phi_{\text {agg }, 0} / D_{a g g}^{3}$, where $\phi_{a g g, 0}$ is the undensified aggregate solids fraction. As in previous studies (Usher et al., 2009), $\phi_{a g g, 0}$ is taken to equal 0.1667 here. As time proceeds, $\phi_{a g g}$ increases from $\phi_{a g g, 0}$ at $t=0$ to a value $\phi_{a g g, \infty}$ (defined as $\phi_{\text {agg }, 0} / D_{a g g, \infty}^{3}$ ) as $t \rightarrow \infty$. Since $D_{a g g, \infty}=0.9$, it follows that $\phi_{\text {agg }, \infty}=0.2286$.

\subsection{Effects of densification on the compressive yield stress}

Changes in the value of $D_{a g g}$ and $\phi_{a g g}$ imply changes in the gel point $\phi_{g}$ and in the compressive yield stress $P_{y}$ vs $\phi$.

The gel point is the overall solids fraction at which aggregates cease to be isolated from one another, but instead begin to overlap and interact with their neighbours. It follows that the ratio $\phi_{g} / \phi_{\text {agg }}$ must correspond to a fixed packing fraction (taken to be 0.6 here). Hence $\phi_{g}=\phi_{g, 0} / D_{a g g}^{3}$. It is possible also to define a value $\phi_{g, \infty}$ equal to $\phi_{g, 0} / D_{a g g, \infty}^{3}$. For the particular values of 
$\phi_{g, 0}=0.1$ and $D_{a g g, \infty}=0.9$ chosen here, it follows that $\phi_{g, \infty}=0.1372$.

Increasing the value of $\phi_{g}$ in itself requires a change in the function $P_{y}(\phi)$ which must vanish identically for $\phi<\phi_{g}$. Hence there are $\phi$ values between $\phi_{g, 0}$ and $\phi_{g, \infty}$ such that $P_{y}$ is non-zero in the undensified state, but identically zero in the fully densified state. If the initial solids fraction $\phi_{f}$ is chosen somewhere between $\phi_{g, 0}$ and $\phi_{g, \infty}$ the column of unconsolidated material at solids fraction $\phi_{f}$ (referred to in Section 2.1 and sketched in Figure 1) cannot survive forever but must instead vanish once $\phi_{g}=\phi_{f}$. Thereafter the height of the consolidated bed $z_{c}$ is identical ${ }^{3}$ to the height of the suspension $H$.

Another constraint placed on the function $P_{y}$ is that when all the gaps between aggregates are removed as $\phi \rightarrow \phi_{\text {agg }}$ and the aggregates thereby lose their discrete character, the function $P_{y}$ joins up smoothly with the compressive yield stress of a 'uniform' space-filling suspension (Channell et al., 2000) without discrete aggregates. Hence for $\phi>\phi_{a g g}$, the densified compressive yield stress $P_{y}$ matches the undensified one (Usher et al., 2009), and moreover $\partial P_{y} / \partial \phi$ is assumed continuous at $\phi_{a g g}$.

As in Section 2.2, weak and strong gel formulae for yield stress (denoted $P_{w y}$ and $\left.P_{s y}\right)$ are considered, and are chosen to have similar functional forms as equations (7)-(8) but respecting the constraints imposed at $\phi=\phi_{g}$ and $\phi=\phi_{a g g}$. Specifically the weak gel $P_{w y}$ has the same form as equation (7) but with $\phi_{g, 0}$ replaced by $\phi_{g}$, and with values $C_{1}$ and $k_{1}$ replacing $C_{0}$ and $k_{0}$

\footnotetext{
${ }^{3}$ Equation (6) for $z_{c}$ remains valid for $\phi_{g}>\phi_{f}$, but since $\partial P_{y} / \partial \phi$ vanishes for $\phi<\phi_{g}$ it is possible to reset the lower limit of the integral to $\phi_{g}$ instead of $\phi_{f}$.
} 
(all other parameters in (7) remaining unchanged). It turns out that

$$
\begin{gathered}
C_{1}=P_{w y, 0}\left(\phi_{a g g}\right)\left(\frac{\left(b+\phi_{a g g}-\phi_{g}\right)\left(\phi_{c p}-\phi_{a g g}\right)}{\left(\phi_{a g g}-\phi_{g}\right)}\right)^{k_{1}} \\
k_{1}=k_{0}\left(\frac{\phi_{a g g}-\phi_{g}}{\phi_{a g g}-\phi_{g, 0}}\right)\left(\frac{b+\phi_{a g g}-\phi_{g}}{b+\phi_{a g g}-\phi_{g, 0}}\right)\left(\frac{\left(\phi_{a g g}-\phi_{g, 0}\right)^{2}+b\left(\phi_{c p}-\phi_{g, 0}\right)}{\left(\phi_{a g g}-\phi_{g}\right)^{2}+b\left(\phi_{c p}-\phi_{g}\right)}\right) .
\end{gathered}
$$

As time $t \rightarrow \infty, C_{1} \rightarrow 4.8057 \mathrm{~Pa}$ and $k_{1} \rightarrow 10.3633$. Likewise the strong gel $P_{s y}$ has the same form as equation (8) but again with $\phi_{g, 0}$ replaced by $\phi_{g}$, and with $c_{1}$ and $K_{1}$ replacing $c_{0}$ and $K_{0}$ (and all other parameters unchanged).

$$
\begin{gathered}
c_{1}=P_{s y, 0}\left(\phi_{a g g}\right) \frac{\left(b_{1}+\phi_{a g g}-\phi_{g}\right)\left(\phi_{c p}-\phi_{a g g}\right)^{K_{1}}}{\left(\phi_{a g g}-\phi_{g}\right)} \\
K_{1}=K_{0}+\left(\phi_{c p}-\phi_{a g g}\right)\left(-\frac{1}{b_{1}+\phi_{a g g}-\phi_{g, 0}}+\frac{1}{\phi_{a g g}-\phi_{g, 0}}\right. \\
\left.+\frac{1}{b_{1}+\phi_{a g g}-\phi_{g}}-\frac{1}{\phi_{a g g}-\phi_{g}}\right) .
\end{gathered}
$$

As $t \rightarrow \infty, c_{1} \rightarrow 6.4516 \mathrm{~Pa}$ and $K_{1} \rightarrow 10.0335$.

Graphs of the compressive yield stress at different levels of densification (corresponding to different aggregate diameter ratios $D_{a g g}$ ) are plotted in Figure 2: both weak and strong gel formulae are considered. At any given $\phi$, there is a general tendency towards lower $P_{y}$ values with more densification (i.e. lower $D_{\text {agg }}$ ). Figure 2 is plotted on a logarithmic scale, which serves (at any given $D_{\text {agg }}$ ) to highlight the differences between weak and strong gel formulae for $\phi$ values just barely greater than $\phi_{g}$. For rather larger $\phi$ values, there is only limited difference between the weak and strong gel formulae. 


\subsection{Solids fraction at bottom boundary}

By assumption, the compressive yield stress formulae are only affected by densification for $\phi<\phi_{a g g}$. If $\phi>\phi_{a g g}$, equations (7) and (8) are assumed to continue to apply. This has implications for the behaviour of $\phi_{b e}$ (the solids fraction at the bottom boundary) defined by equation (5). In order to explain this, two critical solids volumes (per unit cross section) $V_{s}^{\text {upper }}$ and $V_{s}^{\text {lower }}$ are defined as follows

$$
\begin{aligned}
& V_{s}^{\text {upper }}=P_{y, 0}\left(\phi_{\text {agg }, \infty}\right) /(\Delta \rho g) \\
& V_{s}^{\text {lower }}=P_{y, 0}\left(\phi_{\text {agg }, 0}\right) /(\Delta \rho g),
\end{aligned}
$$

where $P_{y, 0}$ is the undensified yield stress, which may follow either the weak or strong gel formulae. These two critical solids volumes are interpreted as follows. If the volume of solids in the suspension $V_{s} \equiv \phi_{f} H_{i}$ is greater than $V_{s}^{u p p e r}$, then $\phi_{b e}$ exceeds $\phi_{a g g, \infty}$, and hence exceeds $\phi_{a g g}$ at all times. It follows from equation (5) that $\phi_{b e}$ is constant with time (because densification does not affect the compressive yield stress when $\phi>\phi_{a g g}$ ). If $V_{s}$ is between $V_{s}^{\text {lower }}$ and $V_{s}^{\text {upper }}$ then $\phi_{b e}$ is between $\phi_{a g g, 0}$ and $\phi_{a g g, \infty}$. This means $\phi_{b e}$ exceeds $\phi_{a g g}$ at early times (implying $\phi_{b e}$ is constant with time during this period), but $\phi_{\text {agg }}$ eventually becomes larger than $\phi_{b e}$. In order to continue to satisfy equation (5), $\phi_{b e}$ must then start to increase with time (these increases in $\phi_{b e}$ compensating for decreases in $P_{y}$ at any given $\phi$ ). Finally if $V_{s}$ is less than $V_{s}^{\text {lower }}$ then $\phi_{b e}$ is less than $\phi_{a g g}$ and moreover is increasing at all times: there is no period when $\phi_{b e}$ is initially constant.

Values of $V_{s}^{\text {upper }}$ and $V_{s}^{\text {lower }}$ for two different suspension rheologies (the 
weak and strong gels) are shown in Table 1.

\subsection{Case studies}

As has been explained in Section 2.4, aggregate densification gives increased $\phi_{g}$ values, but reduced values of $P_{y}$ at any given $\phi$ (at least for $\left.\phi \leq \phi_{\text {agg }}\right)$. This produces changes in the (pseudo-steady state) suspension structure determined by the methods described in Section 2.1. In particular, aggregate densification leads to additional consolidation over and above what occurs during settling of an undensified suspension: see Figure 1.

In what follows the effects of densification are explored via a number of case studies, as outlined in Table 2. Odd numbered cases correspond to the weak gel formula, while even numbered cases correspond to the strong gel formula. Cases 1-6 all have $\phi_{f}=0.105$. This exceeds $\phi_{g, 0}=0.1$ but is less than $\phi_{g, \infty}=0.1372$ : the implication is that the column of unconsolidated material will disappear at some point during the process, leaving only a consolidated bed and a clear liquor zone. This in fact occurs at a critical time $t=0.1759 A^{-1}$. Cases $1-2$ correspond to a small solids volume $V_{s}$ (which is less than $\left.V_{s}^{\text {lower }}\right)$, Cases 3-4 correspond to an intermediate solids volume $V_{s}$ (between $V_{s}^{\text {lower }}$ and $V_{s}^{\text {upper }}$ ) and Cases 5-6 correspond to a large solids volume $V_{s}$ (greater than $V_{s}^{\text {upper }}$ ). Cases 7-8 have the same initial suspension height as Cases 3-4, but with a larger $V_{s}$ owing to having a higher initial solids fraction $\phi_{f}=0.14$. This exceeds $\phi_{g, \infty}$, implying a column of unconsolidated material survives indefinitely. 


\subsection{Converting to dimensionless form}

It is convenient to analyse the model described in Sections 2.1-2.6 in dimensionless form.

Times $t$ are made dimensionless on the scale $A^{-1}$. Dimensionless time will be denoted $T$. Where applicable, the symbol $T_{g, c}$ is used to denote the critical time at which $\phi_{g}$ equals $\phi_{f}$. For Cases $1-6, T_{g, c}=0.1759$.

There are two possible scales for converting the distance coordinate $z$ to a dimensionless coordinate $Z$. One such scale is the initial suspension height $H_{i}$. The other follows from the rheology, e.g. $C_{0} /(\Delta \rho g)$ where $C_{0}$ is the parameter appearing in the formula for $P_{w y, 0}$. This latter scale is likely to be more convenient for comparison with experimental batch settling data, as the definition of $Z$ then remains the same for a series of experiments done on a given suspension at various different initial heights.

In a similar fashion, it is possible to define a dimensionless bed height $Z_{c}$ (in lieu of $z_{c}$ ) and a dimensionless suspension height $L$ (in lieu of $H$ ). Moreover $Z_{c, 0}, Z_{c, \infty}, L_{0}$ and $L_{\infty}$ are defined to be undensified and fully densified analogues of $Z_{c}$ and $L$ respectively.

Note that the ratio between $H_{i}$ and $C_{0} /(\Delta \rho g)$ is a dimensionless group (the dimensionless initial suspension height). It is denoted by the symbol $L_{i}$. The dimensionless coordinate $Z$ and the dimensionless heights $Z_{c}$, L, etc. can be normalised by dividing through by $L_{i}$, this then being equivalent to normalising dimensional distances by $H_{i}$. 


\section{Results and discussion}

Section 3 broadly follows the structure used in the original manuscript. Changes have been highlighted in colour.

Comparisions between suspension heights and consolidated bed heights obtained before and after aggregate densification, and the associated solids fractions attained, are the main results of interest here (see Section 3.1). The evolutions of the suspension height and the consolidated bed height are also considered (see Section 3.2), as are the evolutions of the consolidated bed structures (see Section 3.3).

\subsection{Values of $\phi_{b e}, Z_{c}$ and $L$ before and after densification}

Table 3 shows that, for an initially networked system where there is no aggregate densification occurring, the solids volume fraction obtained at the bottom of the suspension $\phi_{b e, 0}$ increases with an increase of the initial suspension height ${ }^{4}$ for a specified initial feed solids volume fraction, $\phi_{f}$. Moreover $\phi_{b e, 0}$ also increases with increasing $\phi_{f}$. Once aggregate densification occurs, the solids volume fraction obtained at the bottom of the batch settler, $\phi_{b e}$ can increase over and above $\phi_{b e, 0}$, but only in Cases 1-4 for which $\phi_{b e, 0}$ is not already so large as to exceed $\phi_{a g g, \infty}$. Moreover in Cases 3-4 for which $\phi_{b e, 0}$ is greater than $\phi_{a g g, 0}$, it turns out that $\phi_{b e}$ only starts increase once $\phi_{a g g}$ exceeds $\phi_{b e, 0}$. This was found to occur at time $T=2.591$ for the weak gel formula (Case 3) and $T=2.598$ for the strong gel formula (Case 4).

Table 3 also presents the heights of the suspension and the consolidated

\footnotetext{
${ }^{4}$ Reflecting this change in the solids volume fraction, the settled suspension height relative to the initial suspension height undergoes (in Table 3) a corresponding decrease.
} 
bed predicted at both the initially undensified and fully densified states. The heights of the suspension in the fully densified state are invariably smaller than their undensified counterparts. Nevertheless the heights of the consolidated bed predicted at the fully densified equilibrium state are taller than those determined at the undensified equilibrium state for Cases 7-8 (contrast with the bed heights in e.g. Cases 1-6). This is due to a larger initial feed solids volume fraction, $\phi_{f}$ having been given in Cases 7-8 leading to a quite significant amount of mass in an unconsolidated column retained above the consolidated bed for an undensified system (most of this mass then entering the consolidated bed in the fully densified system).

For Cases 1-6, the suspension heights and the consolidated bed heights calculated in the fully densified equilibrium state batch settler are equal to one another (i.e. no unconsolidated column of material survives) since the initial feed solids volume fraction, $\phi_{f}$ is smaller than the fully densified gel point, $\phi_{g, \infty}$. Although the consolidated bed heights determined in the initially undensified equilibrium state batch settler using the weak gel formula are taller than those using the strong gel formula (e.g. odd vs. even numbered cases), the suspension heights determined in the undensified equilibrium state batch settler using the weak gel formula are smaller, due to the differing behaviours of the compressive yield stress formulae evaluated at or near $\phi_{f}$ when $\phi_{f}$ is itself not too far from $\phi_{g}$.

Despite these differences just noted, the (undensified) suspension heights and the consolidated bed heights calculated using the weak gel formula are broadly similar compared to those determined using the strong gel formula in Cases 7-8, which have a larger $\phi_{f}$, due to comparatively good agreement 
of the strong and weak gel functional forms over much of the local solids volume fraction domain (i.e. away from the neighbourhood of the gel point; the solids volume fractions everywhere being a finite amount above the gel point in Cases 7-8).

\subsection{Evolutions of the heights of the suspension and the consolidated bed}

Figure 3-Figure 6 show the evolutions (as predicted via the pseudo-steady state model) of the heights of the suspension and the consolidated bed for Cases 1-8. For Cases 1-6, the suspension heights intersect the consolidated bed heights at a critical dimensionless time (here equal to 0.1759 as mentioned earlier), since the initial feed solids volume fraction, $\phi_{f}$ is smaller than the fully densified gel point, $\phi_{g, \infty}$. After this critical time for Cases 1-6, the consolidated bed heights and suspension heights coincide and then decrease until the fully densified equilibrium state is obtained. On the other hand, for Cases $7-8$, the consolidated bed heights are always less than the suspension heights, due to a larger specified initial feed solids volume fraction, $\phi_{f}$ that exceeds the fully densified point, $\phi_{g, \infty}$.

The functional form of the compressive yield stress affects the evolution of the consolidated bed height significantly for Cases 1-8. Figure 3-Figure 5 show that the consolidated bed heights determined in Cases 1-6 always decrease for densification time, $T$ larger than the critical time, $T_{g, c}$, since the materials experience more dewatering as they approach the fully densified equilibrium state. As also shown in Figure 3-Figure 5, the consolidated bed heights determined for those cases using the strong gel formula always increase until the densification time, $T$ equals the critical time, $T_{g, c}$ (e.g. 
Cases 2, 4, and 6) whilst the consolidated bed heights determined for the cases using the weak gel formula increase up to a maximum value at some time that is earlier than the critical time, $T_{g, c}$, and then decrease thereafter (e.g. Cases 1, 3, and 5). The reason for observing the decreases in the (weak gel) consolidated bed heights for $T<T_{g, c}$ in Figure 3-Figure 5 is due to the very poor weight bearing abilities of the weak gel for the solids volume fractions close to the gel point. This is reflected in the amount of material that remains in the unconsolidated column. Equation (3) indicates that this is proportional to $P_{y}\left(\phi_{f}\right)$, a function which collapses very dramatically in the weak gel case as the gel point, $\phi_{g}$ approaches the initial feed solids volume fraction, $\phi_{f}$. Indeed when the suspension heights and the consolidated bed heights meet in Figure 3-Figure 5, they do so tangentially for weak gels, reflecting the fact that the unconsolidated layer has collapsed so as to be exceedingly thin for quite some time on the approach to $T_{g, c}$.

The feature (in the weak gel case) of having a maximum in the consolidated bed height prior to the suspension height and the consolidated bed height coinciding is specific to slowly densifying systems. For the opposite limit of rapid densification, in which a suspension might switch from being gelled to ungelled, and attain full densification even before significant settling has occurred, this feature is unlikely to be observed. That limit corresponds instead to a conventional batch settling problem but starting from an unnetworked system, in which consolidated bed height grows, up until the point that it coincides with the suspension height: the bed height will then decrease after this point, but not beforehand. Thus, the qualitative curve shapes plotted in Figure 3-Figure 5 using the strong gel formula with 
slow densification might be also observed when densification is more rapid. However, the curve shapes plotted in Figure 3-Figure 5 using the weak gel formula and slow densification (which exhibit a decrease in the consolidated bed height even whilst the unconsolidated suspension above the bed remains gelled) differ qualitatively from their rapid densification counterparts. It is not clear whether it is possible to observe that predicted decrease in the consolidated bed height in experiments. One might anticipate that these effects on the consolidated bed height could be observed experimentally, although the consolidation zone cannot always be readily visualised in experiments: rather it is the top of the suspension that is more easily detected.

For Cases 7-8, the consolidated bed heights always increase until the fully densified equilibrium state is achieved $^{5}$. As shown in Figure 6, near the fully densified state, when the weak gel formula is used to predict the heights of the suspension and the consolidated bed, the difference between these two heights (being itself the height of the unconsolidated column) is smaller, compared to that obtained using the strong gel formula, since the compressive yield stress determined at $\phi=\phi_{f}$ using the weak gel formula is substantially smaller than that determined using the strong gel formula.

Although the graphs for the consolidated bed height and the suspension height given in Figure 3-Figure 6 show clearly how the system responds to aggregate densification, there is another aspect of these figures which is worth mentioning. In Figure 3, sedimentation (on a rapid time scale that is left unresolved here) takes the system from an initial normalised height of

\footnotetext{
${ }^{5}$ The final time $T=10$ shown in Figure 6 effectively gives full densification, with an aggregate diameter ratio $D_{\text {agg }}$ differing from $D_{\text {agg }, \infty} \equiv 0.9$ by only $5 \times 10^{-6}$.
} 
unity down to 0.78 , whereas densification only takes it from the normalised height of 0.78 down to roughly 0.68. Moreover, Figure 4-Figure 6 show even more modest benefits from densification. Even though dewatering is clearly enhanced by aggregate densification, for these data at least, much of the dewatering was already achieved during the initial settling phase before the onset of densification.

\subsection{Consolidated bed structure}

Figure 7-Figure 10 show the solids volume fraction profiles $\phi$ vs $Z$ for the suspension at various different dimensionless times $T$. The solids volume fractions predicted in the consolidation zone increase moving downwards into the consolidated bed as shown in Figure 7-Figure 10. When the local solids volume fraction, $\phi$ is larger than the solids volume fraction within the aggregates, $\phi_{a g g}$, the densified compressive yield stress functional form is unchanged from the undensified one. Thus, the same slope of the curves must be observed, at least low down in the consolidated bed, as is particularly noticeable for Figure 9-Figure 10 (i.e. Cases 5-8). If the initial suspension is very tall (e.g. Cases 5-6) or the initial suspension has a comparatively high solids volume fraction (e.g. Cases 7-8), undensified sludge rheological properties actually continue to apply in quite a significant part of the consolidated bed, thus there is only very marginal benefit of densification.

What is perhaps slightly more surprising is that Figure 8 indicates that Cases 3-4 show a comparatively modest benefit from densification also. Here the solids volume fraction profiles are only constrained to have the same slope low down in the suspension for early times such that parts of the 
suspension still have $\phi>\phi_{\text {agg }}$. The point in the suspension at which $\phi=\phi_{\text {agg }}$ however migrates downward, reaching the bottom boundary at a finite time ( $T=2.591$ for Case 3 , and $T=2.598$ for Case 4$)$. After that, the slope of the $\phi$ vs $Z$ profile and indeed the value of solids fraction itself at the bottom boundary $\phi_{b e}$ are no longer constrained to their original values. However the change in $\phi_{b e}$ is very tiny indeed (as Table 3 makes apparent) and not easily seen on the scale of Figure 8.

Now consider the situation near the top of the consolidated bed in Figure 7-Figure 10. When predicted using the weak gel formula (once the unconsolidated zone has already disappeared), locally flat segments of the curves are observed (in e.g. Cases 1, 3, and 5) at the top of the consolidated bed. This is due to both the compressive yield stress and the derivative of the compressive yield stress with respect to the local solids volume fraction determined using the weak gel formula approaching zero near the gel point (Zhang et al., 2013b). The implication is that the solids volume fraction obtained near the top of the consolidated bed changes significantly as the precise position in the consolidated bed changes slightly (Zhang et al., 2013b). This corroborates the statements made (see Section 3.2) about local collapse of the consolidated bed near the gel point. A similar behaviour is also predicted in Zhang et al. (2013b), i.e. values of solids fraction close to $\phi_{g}$ are collapsed into an exceedingly small height range.

\section{Conclusions}

In an initially networked batch setter where slow aggregate densification occurs, the pseudo-steady state model has been used to predict the evolu- 
tions of the heights of the suspension and the consolidated bed, and the consolidated bed structures. Cases were considered where the initial feed solids fraction $\phi_{f}$ exceeded the initial suspension gel point $\phi_{g, 0}$ for undensified systems. For $\phi_{f}$ less than the fully densified gel point, $\phi_{g, \infty}$, different compressive yield stress functional forms (i.e. weak vs strong gel formulae) can affect the evolutions of the consolidated bed heights with marked differences for times immediately before the critical time at which the suspension height and the consolidated bed height intersect. For $\phi_{f}$ greater than $\phi_{g, \infty}$ however, these two heights never intersect, i.e. a column of unconsolidated material (with solids fraction remaining at $\phi_{f}$ ) persists indefinitely.

The consolidated bed height determined at the fully densified equilibrium state is typically shorter than that calculated at the undensified equilibrium state for $\phi_{f}<\phi_{g, \infty}$ but can be taller than that determined at the undensified equilibrium state for $\phi_{f}>\phi_{g, \infty}$ : this reflects a significantly higher amount of solids (in the undensified case compared to the densified one) that are retained in an unconsolidated column.

Aggregate densification can also affect the consolidated bed structure. One way this manifests itself is in the value of solids volume fraction at the bottom of the batch settler $\phi_{b e}$. It is possible to have either some increase or no increase whatsoever in $\phi_{b e}$ after aggregate densification, the former scenario occurring if the initial suspension height, $H_{i}$ is comparatively short and/or the initial feed solids volume fraction, $\phi_{f}$ is comparatively small, either scenario implying that the total volume of solids $V_{s}$ is low. Increasing $V_{s}$ leads to less change (or indeed no change at all) in $\phi_{b e}$ as the aggregates densify. The solids fraction profile low down in the bed is also insensitive 
to densification in these latter cases. Although aggregate densification is generally rather beneficial for dewatering, in these latter cases mentioned, the benefits can actually be somewhat marginal.

\section{Acknowledgements}

PG acknowledges sabbatical stay funding from CONICET Argentina res. no. $2218 / 13$.

\section{References}

Berres, S., Bürger, R., Coronel, A., Sepulveda, M., 2005. Numerical identification of parameters for a strongly degenerate convection-diffusion problem modelling centrifugation of flocculated suspensions. Appl. Numer. Math. $52,311-337$.

Bürger, R., Bustos, M. C., Concha, F., 1999. Settling velocities of particulate systems: 9. Phenomenological theory of sedimentation processes: Numerical simulation of the transient behavior of flocculated suspensions in an ideal batch or continuous thickener. International Journal of Minerals Processing 55 (4), 267-282.

Bürger, R., Concha, F., 1998. Mathematical model and numerical simulation of the settling of flocculated suspensions. International Journal of Multiphase Flow 24, 1005-1023.

Buscall, R., White, L. R., 1987. The consolidation of concentrated suspensions: Part 1. The theory of sedimentation. J. Chem. Soc. - Faraday Trans. $83,873-891$. 
Channell, G. M., Miller, K. T., Zukoski, C. F., 2000. Effects of microstructure on the compressive yield stress. AIChE Journal 46, 72-78.

Channell, G. M., Zukoski, C. F., 1997. Shear and compressive rheology of aggregated alumina suspensions. AIChE Journal 43, 1700-1708.

de Kretser, R. G., Usher, S. P., Scales, P. J., Boger, D. V., Landman, K. A., 2001. Rapid filtration measurement of dewatering design and optimisation parameters. AIChE Journal 47, 1758-1769.

Diehl, S., 2007. Estimation of the batch-settling flux function for an ideal suspension from only two experiments. Chemical Engineering Science 62, 4589-4601.

Farrow, J. B., Johnston, R. R. M., Simic, K., Swift, J. D., 2000. Consolidation and aggregate densification during gravity thickening. Chemical Engineering Journal 80, 141-148.

Gladman, B., de Kretser, R. G., Rudman, M., Scales, P. J., 2005. Effect of shear on particulate suspension dewatering. Chemical Engineering Research and Design 83, 933-936.

Gladman, B. R., 2004. The effect of shear on dewatering of flocculated suspensions. Ph.D. thesis, University of Melbourne, Melbourne, Australia.

Gladman, B. R., Rudman, M., Scales, P. J., 2010. The effect of shear on gravity thickening: Pilot scale modelling. Chemical Engineering Science $65,4293-4301$. 
Grassia, P., Usher, S. P., Scales, P. J., 2008. A simplified parameter extraction technique using batch settling data to estimate suspension material properties in dewatering applications. Chemical Engineering Science 63, $1971-1986$.

Grassia, P., Usher, S. P., Scales, P. J., 2011. Closed-form solutions for batch settling height from model settling flux functions. Chemical Engineering Science 66, 964-972.

Grassia, P., Zhang, Y., Martin, A. D., Usher, S. P., Scales, P. J., Crust, A., Spehar, R., 2014. Effects of aggregate densification upon thickening of Kynchian suspensions. Chemical Engineering Science 111, 56-72.

Green, M. D., Eberl, M., Landman, K. A., 1996. Compressive Yield Stress of Flocculated Suspensions: Determination via Experiment. AIChE Journal $42,2308-2318$.

Green, M. D., Landman, K. A., de Kretser, R. G., Boger, D. V., 1998. Pressure filtration technique for complete characterization of consolidating suspensions. Ind. Eng. Chem. Res. 37, 4152-4156.

Howells, I., Landman, K. A., Panjkov, A., Sirakoff, C., White, L. R., 1990. Time-dependent batch settling of flocculated suspensions. Appl. Math. Modelling 14, 77-86.

Kynch, G. J., 1952. A theory of sedimentation. Transactions of the Faraday Society 48, 166-176. 
Landman, K. A., White, L. R., 1994. Solid/liquid separation of flocculated suspensions. Advances in Colloid and Interface Science 51, 175-246.

Landman, K. A., White, L. R., 1997. Predicting filtration time and maximizing throughput in a pressure filter. AIChE Journal 43, 3147-3160.

Landman, K. A., White, L. R., Buscall, R., 1988. The continuous-flow gravity thickener: Steady state behavior. AIChE Journal 34, 239-252.

Landman, K. A., White, L. R., Eberl, M., 1995. Pressure filtration of flocculated suspensions. AIChE Journal 41, 1687-1700.

Lester, D. R., Usher, S. P., Scales, P. J., 2005. Estimation of the Hindered Settling Function $\mathrm{R}(\phi)$ from Batch-Settling Tests. AIChE Journal 51, 11581168.

Martin, A. D., 2004. Optimisation of clarifier-thickeners processing stable suspensions for turn-up/turn-down. Water Research 38, 1568-1578.

Spehar, R., Kiviti-Manor, A., Fawell, P., Usher, S. P., Rudman, M., Scales, P. J., 2014. Aggregate densification in the thickening of flocculated suspensions in an un-networked bed. Chemical Engineering Science 122, 585-595.

Stickland, A. D., Burgess, C., Dixon, D. R., Harbour, P. J., Scales, P. J., Studer, L. J., Usher, S. P., 2008. Fundamental dewatering properties of wastewater treatment sludges from filtration and sedimentation testing. Chemical Engineering Science 63, 5283-5290.

Stickland, A. D., White, L. R., Scales, P. J., 2006. Modelling of solid-bowl 
batch centrifugation of flocculated suspensions. AIChE Journal 52, 13511362.

Usher, S. P., de Kretser, R. G., Scales, P. J., 2001. Validation of a New Filtration Technique for Dewaterability Characterization. AIChE Journal $47,1561-1570$.

Usher, S. P., Scales, P. J., 2005. Steady state thickener modelling from the compressive yield stress and hindered settling function. Chemical Engineering Journal 111, 253-261.

Usher, S. P., Spehar, R., Scales, P. J., 2009. Theoretical analysis of aggregate densification: Impact on thickener performance. Chemical Engineering Journal 151, 202-208.

van Deventer, B. B. G., 2012. Aggregate Densification Behaviour in Sheared Supensions. Ph.D. thesis, University of Melbourne, Melbourne, Australia.

van Deventer, B. B. G., Usher, S. P., Kumar, A., Rudman, M., Scales, P. J., 2011. Aggregate densification and batch settling. Chemical Engineering Journal 171, 141-151.

Zhang, Y., Martin, A., Grassia, P., 2013a. Mathematical modelling of timedependent densified thickeners. Chemical Engineering Science 99, 103-112.

Zhang, Y., Martin, A., Grassia, P., 2013b. Prediction of thickener performance with aggregate densification. Chemical Engineering Science 101, 346-358. 


\begin{tabular}{|l|l|l|}
\hline Gel formula & $V_{s}^{\text {lower }} / \mathrm{m}$ & $V_{s}^{\text {upper }} / \mathrm{m}$ \\
\hline Weak & 0.01624 & 0.05891 \\
\hline Strong & 0.01603 & 0.05890 \\
\hline
\end{tabular}

Table 1: Values of critical solids volumes (per unit cross section) $V_{s}^{\text {lower }}$ and $V_{s}^{\text {upper }}$ for different forms of the compressive yield stress (weak vs strong gel formulae). The solids fraction at the bottom boundary of the suspension evolves differently according to whether the volume of solids in the suspension $V_{s}$ is greater than $V_{s}^{\text {upper }}$, between $V_{s}^{\text {lower }}$ and $V_{s}^{\text {upper }}$, or less than $V^{\text {lower }}$. 


\begin{tabular}{|l|l|l|l|l|}
\hline & Gel formula & $\phi_{f}$ & $H_{i} / \mathrm{m}$ & $V_{s} / \mathrm{m}$ \\
\hline Case 1 & Weak & 0.105 & 0.15 & 0.01575 \\
\hline Case 2 & Strong & 0.105 & 0.15 & 0.01575 \\
\hline Case 3 & Weak & 0.105 & 0.5 & 0.0525 \\
\hline Case 4 & Strong & 0.105 & 0.5 & 0.0525 \\
\hline Case 5 & Weak & 0.105 & 0.8 & 0.084 \\
\hline Case 6 & Strong & 0.105 & 0.8 & 0.084 \\
\hline Case 7 & Weak & 0.14 & 0.5 & 0.07 \\
\hline Case 8 & Strong & 0.14 & 0.5 & 0.07 \\
\hline
\end{tabular}

Table 2: Parameters used for case studies, including different forms of the compressive yield stress (i.e. weak vs strong gel formulae), initial feed solids fraction $\phi_{f}$, initial suspension height $H_{i}$, and the volume of solids (per unit cross section) $V_{s}$, which is $\phi_{f} H_{i}$. For comparison with the $\phi_{f}$ values, the undensified gel point is $\phi_{g, 0}=0.1$ and the gel point in the fully densified state is $\phi_{g, \infty}=0.1372$. 


\begin{tabular}{|l|l|l|l|l|}
\hline & $\phi_{b e, 0}$ & $\phi_{b e, \infty}$ & $\phi_{\text {agg }, 0}$ & $\phi_{\text {agg }, \infty}$ \\
\hline Case 1 & 0.1653 & 0.1725 & 0.1667 & 0.2286 \\
\hline Case 2 & 0.1659 & 0.1723 & 0.1667 & 0.2286 \\
\hline Case 3 & 0.22305 & 0.22308 & 0.1667 & 0.2286 \\
\hline Case 4 & 0.22309 & 0.22312 & 0.1667 & 0.2286 \\
\hline Case 5 & 0.2458 & 0.2458 & 0.1667 & 0.2286 \\
\hline Case 6 & 0.2457 & 0.2457 & 0.1667 & 0.2286 \\
\hline Case 7 & 0.2370 & 0.2370 & 0.1667 & 0.2286 \\
\hline Case 8 & 0.2370 & 0.2370 & 0.1667 & 0.2286 \\
\hline \hline & $Z_{c, 0} / L_{i}$ & $L_{0} / L_{i}$ & $Z_{c, \infty} / L_{i}$ & $L_{\infty} / L_{i}$ \\
\hline Case 1 & 0.767 & 0.780 & 0.671 & 0.671 \\
\hline Case 2 & 0.717 & 0.787 & 0.682 & 0.682 \\
\hline Case 3 & 0.601 & 0.605 & 0.569 & 0.569 \\
\hline Case 4 & 0.586 & 0.606 & 0.572 & 0.572 \\
\hline Case 5 & 0.543 & 0.5455 & 0.523 & 0.523 \\
\hline Case 6 & 0.533 & 0.5465 & 0.525 & 0.525 \\
\hline Case 7 & 0.6196 & 0.739 & 0.720 & 0.721 \\
\hline Case 8 & 0.6195 & 0.738 & 0.7035 & 0.7235 \\
\hline
\end{tabular}

Table 3: The predictions of solids volume fractions determined at the bottom of the batch settler $\phi_{b e}$, and heights of the suspension $L$ and of the consolidated bed $Z_{c}$ (normalised by the dimensionless initial suspension height $L_{i}$ ) in both the initially undensified equilibrium state and the fully densified equilibrium state batch settlers. Recall from Table 2, that Cases 1-2 have a shorter initial suspension height than Cases 3-4, whereas Cases 5-6 have a taller initial suspension height. Meanwhile Cases 7-8 have a higher initial feed solids volume fraction than any of the others. Odd numbered cases correspond to weak gels, and even numbered cases correspond to strong gels. 


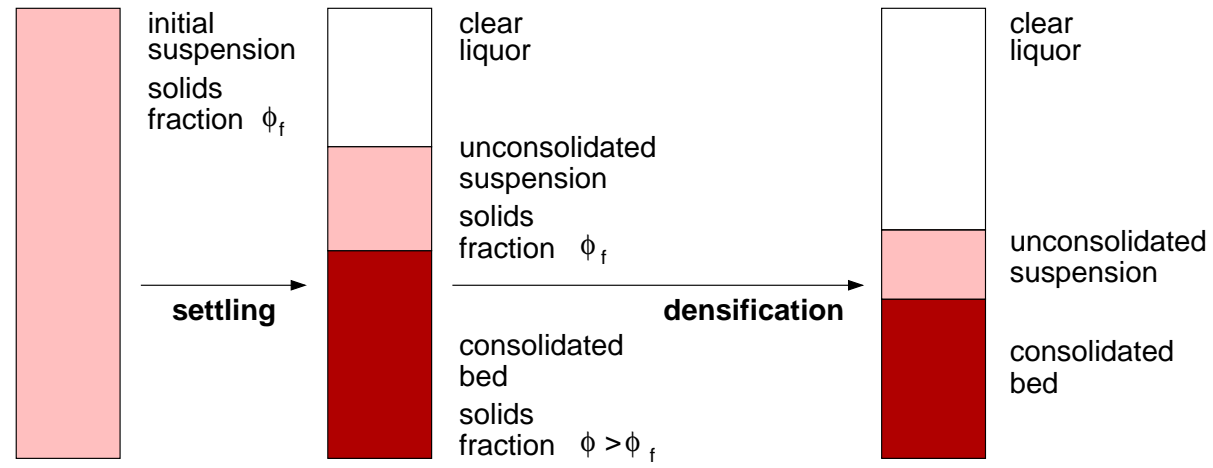

Figure 1: A networked suspension with an initial feed solids fraction $\phi_{f}$ settles into a consolidated bed (below) and a clear liquor zone (above), retaining a column of unconsolidated material between the two. If however, under the action of shear, the suspension undergoes a process of slow aggregate densification, further consolidation (at pseudo-steady state) may occur. The column of unconsolidated material survives only for as long as the suspension's gel point $\phi_{g}$ remains less than $\phi_{f}$. 


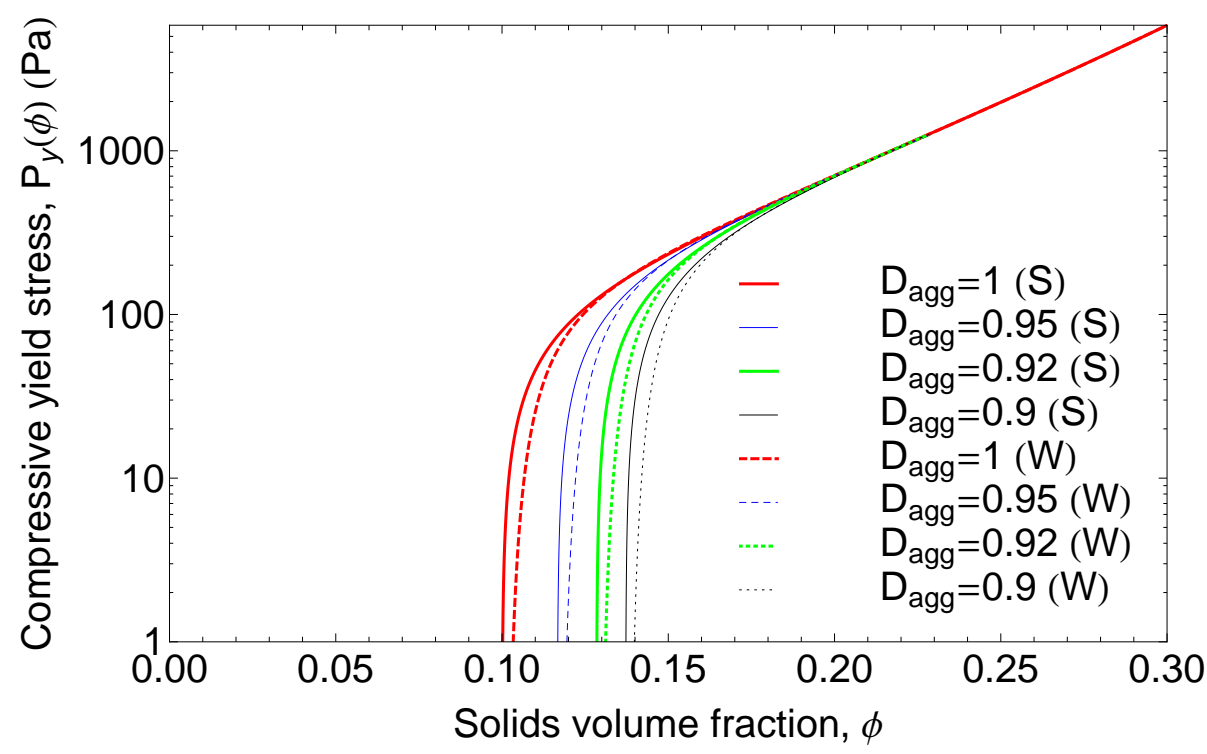

Figure 2: Compressive yield stress curves determined using both the strong gel (S) formula and the weak gel (W) formula with different levels of aggregate densification, represented via an aggregate diameter ratio, $D_{a g g}$. Note that the initially undensified state corresponds to the aggregate diameter ratio, $D_{a g g}=1$ (leftmost curves) and full densification corresponds to the aggregate diameter ratio, $D_{a g g}=0.9$ (rightmost curves). 

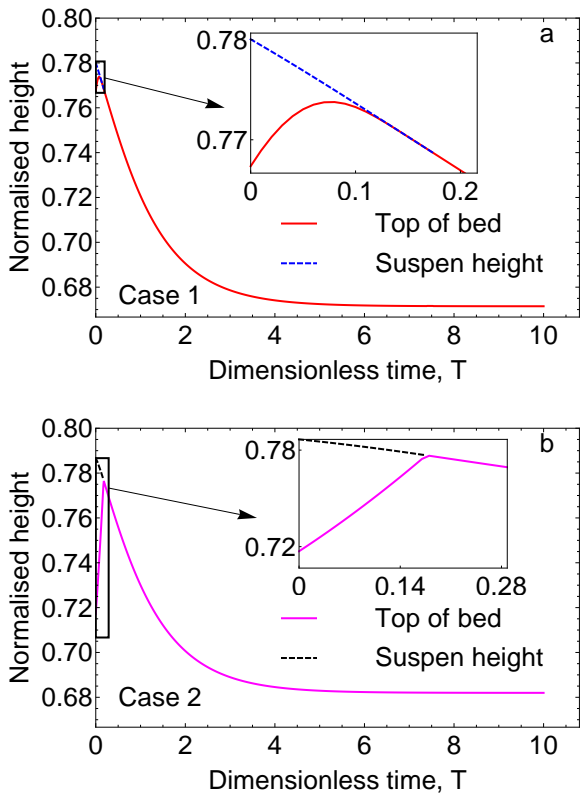

Figure 3: Evolutions of the heights of the suspension and the consolidated bed for Cases 12 (both normalised by the dimensionless initial suspension height, $L_{i}$ ). The time axis is measured from the point at which aggregate densification is considered to begin: here aggregate densification is assumed to be a much slower process than batch settling, so the initial suspension settles (effectively instantaneously) in its undensified state, and only then does densification begin. Here the sub-plot labelled 'a' denotes the heights plotted for Case 1 (weak gel) whilst the sub-plot labelled ' $b$ ' represents the heights plotted for Case 2 (strong gel). Recall that the initial feed solids volume fraction, $\phi_{f}$ equals 0.105 in Cases $1-2$, and this is less than the fully densified gel point, $\phi_{g, \infty}$ which equals 0.1372 . Under these circumstances, suspension height and bed height coincide after a critical time $T_{g, c}$ equal to 0.1759 here. 

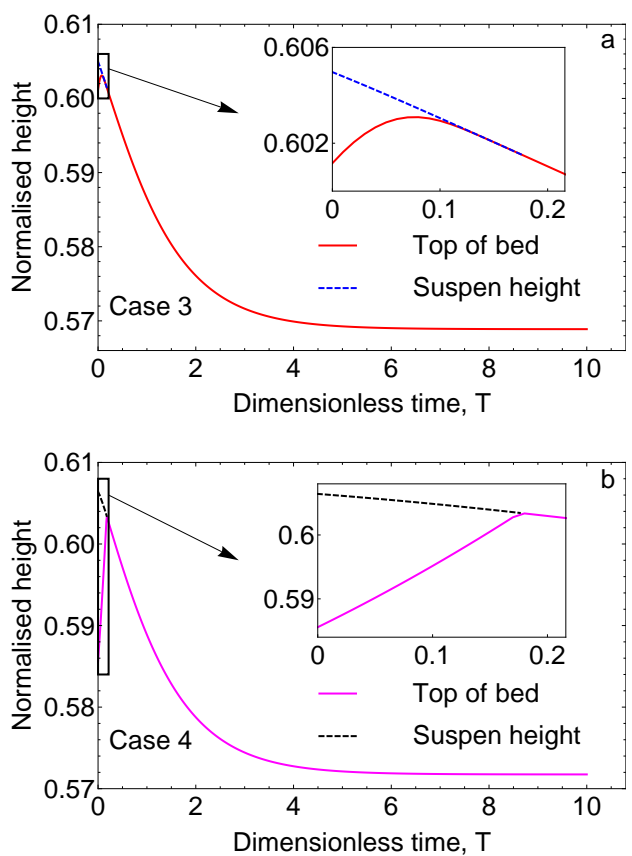

Figure 4: Evolutions of the suspension heights and the consolidated bed heights for Cases 3-4 (both normalised by the dimensionless initial suspension height, $L_{i}$ ). Here the sub-plots labelled 'a' and 'b' denote the heights plotted for Case 3 (weak gel) and Case 4 (strong gel), respectively. 

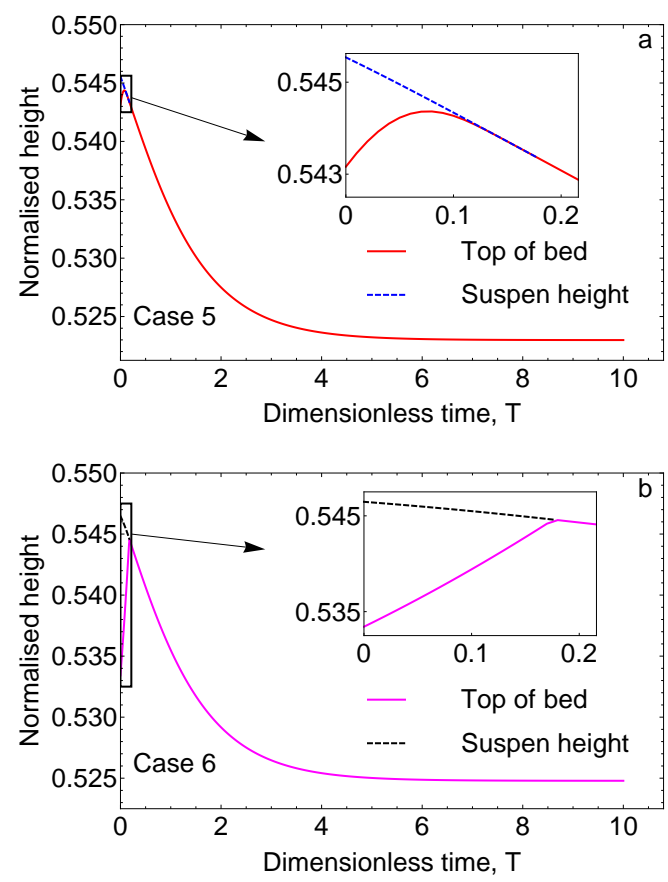

Figure 5: Evolutions of suspension heights and consolidated bed heights for Cases 5-6 (both normalised by the dimensionless initial suspension height, $L_{i}$ ). Case 5 (weak gel) and Case 6 (strong gel) are shown in the sub-plots labelled 'a' and 'b', respectively. 

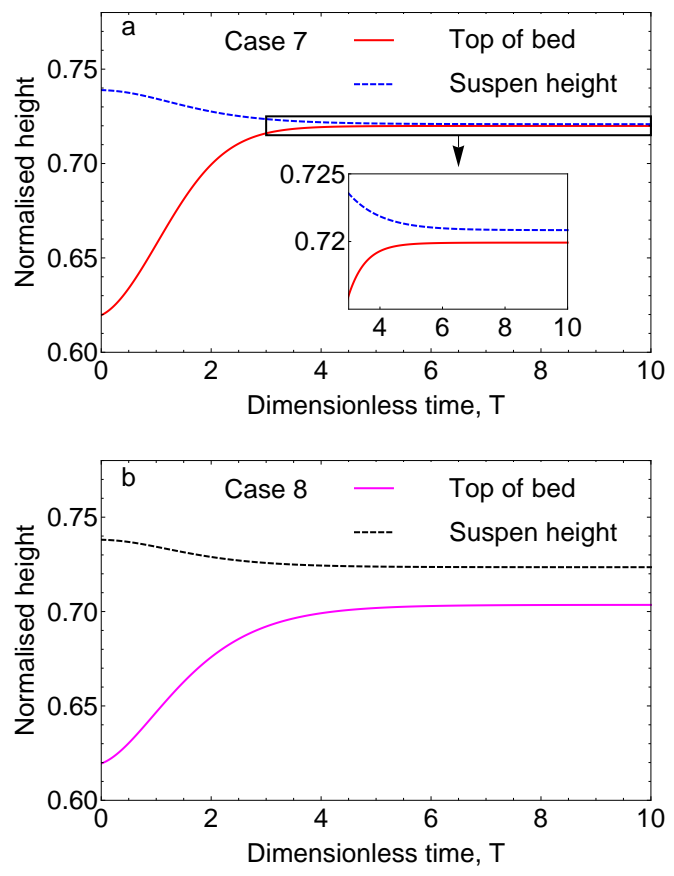

Figure 6: Predictions of the heights of the suspension and the consolidated bed for Cases 78 (both normalised by the dimensionless initial suspension height, $L_{i}$ ). The sub-plot labelled ' $a$ ' represents the heights for Case 7 (weak gel) and the sub-plot labelled ' $b$ ' denotes the heights for Case 8 (strong gel). Recall that the initial feed solids volume fraction, $\phi_{f}$ equals 0.14 in Cases $7-8$, and this is greater than the fully densified gel point, $\phi_{g, \infty}$ which equals 0.1372 . Under these circumstances, the suspension height and bed height never coincide. 

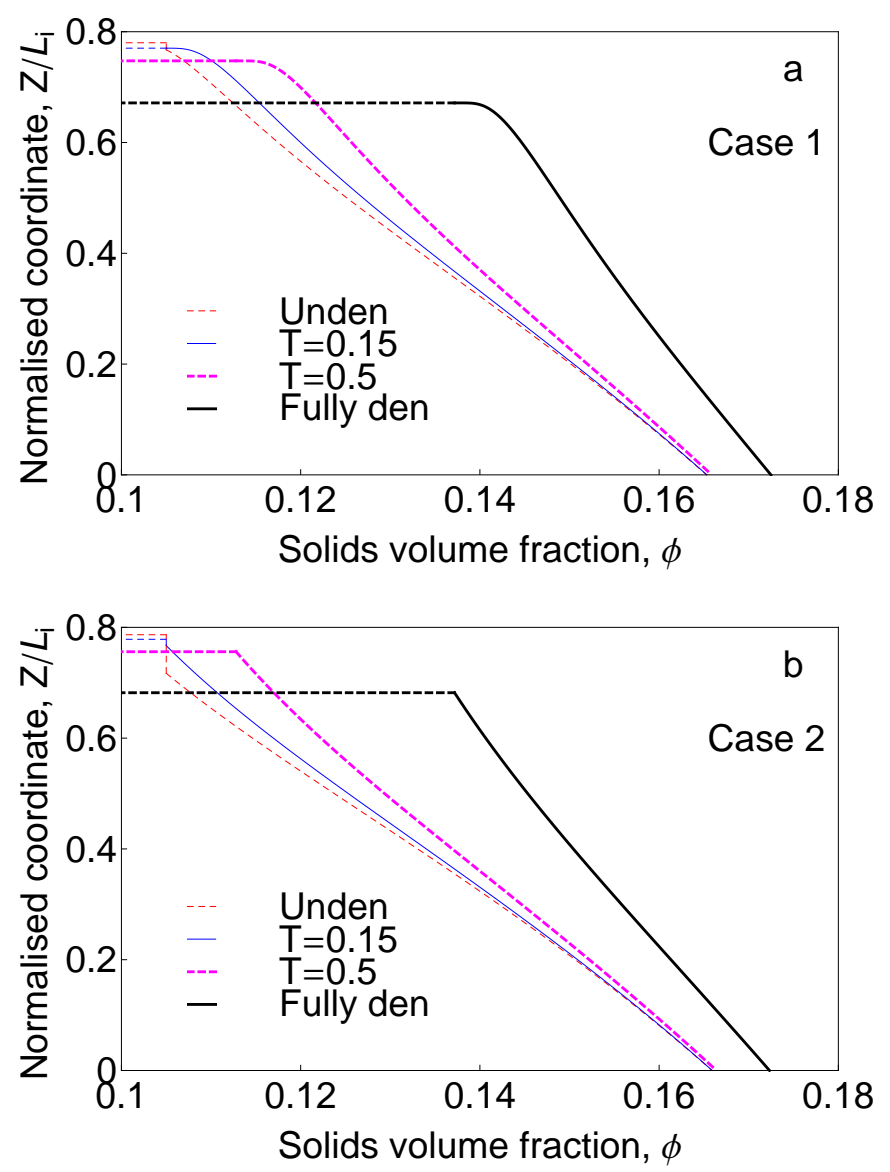

Figure 7: The solids volume fraction profiles predicted for Cases 1-2 for the undensified state, the fully densified state, and intermediate levels of aggregate densification, represented by a densification time $T$. The sub-plot labelled 'a' denotes the consolidated bed structures for Case 1 (weak gel) whilst the sub-plot labelled 'b' represents the consolidated bed structures for Case 2 (strong gel). Recall that the initial feed solids volume fraction, $\phi_{f}$ equals 0.105 in Cases 1-2. At sufficiently early times there can be an unconsolidated column at a uniform solids volume fraction, $\phi_{f}$, although this disappears at later times. 

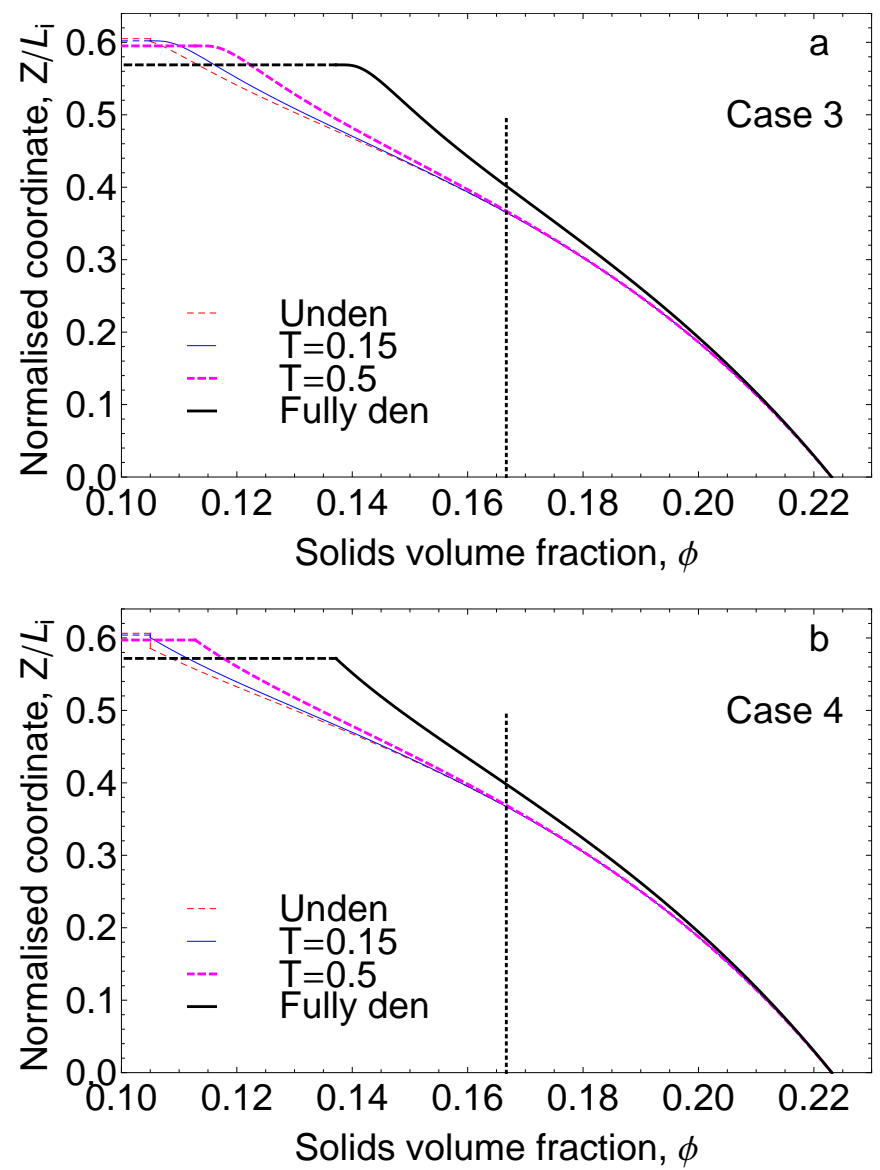

Figure 8: The solids volume fraction profiles determined for Cases 3-4. The sub-plots labelled ' $a$ ' and 'b' denote the consolidated bed structures predicted for Case 3 (weak gel) and Case 4 (strong gel), respectively. The dotted vertical lines represent for $T=0$ (the undensified state), the boundary between those parts of the bed for which $\phi<\phi_{\text {agg }}$ and those parts for which $\phi>\phi_{a g g}$ (which governs whether or not densified suspension properties differ from their undensified counterparts). The boundary migrates downward over time reaching the bottom of the suspension at the particular time when $\phi_{\text {agg }}$ equals $\phi_{b e, 0}$. This occurs when $T=2.591$ in Case 3 and $T=2.598$ in Case 4. 

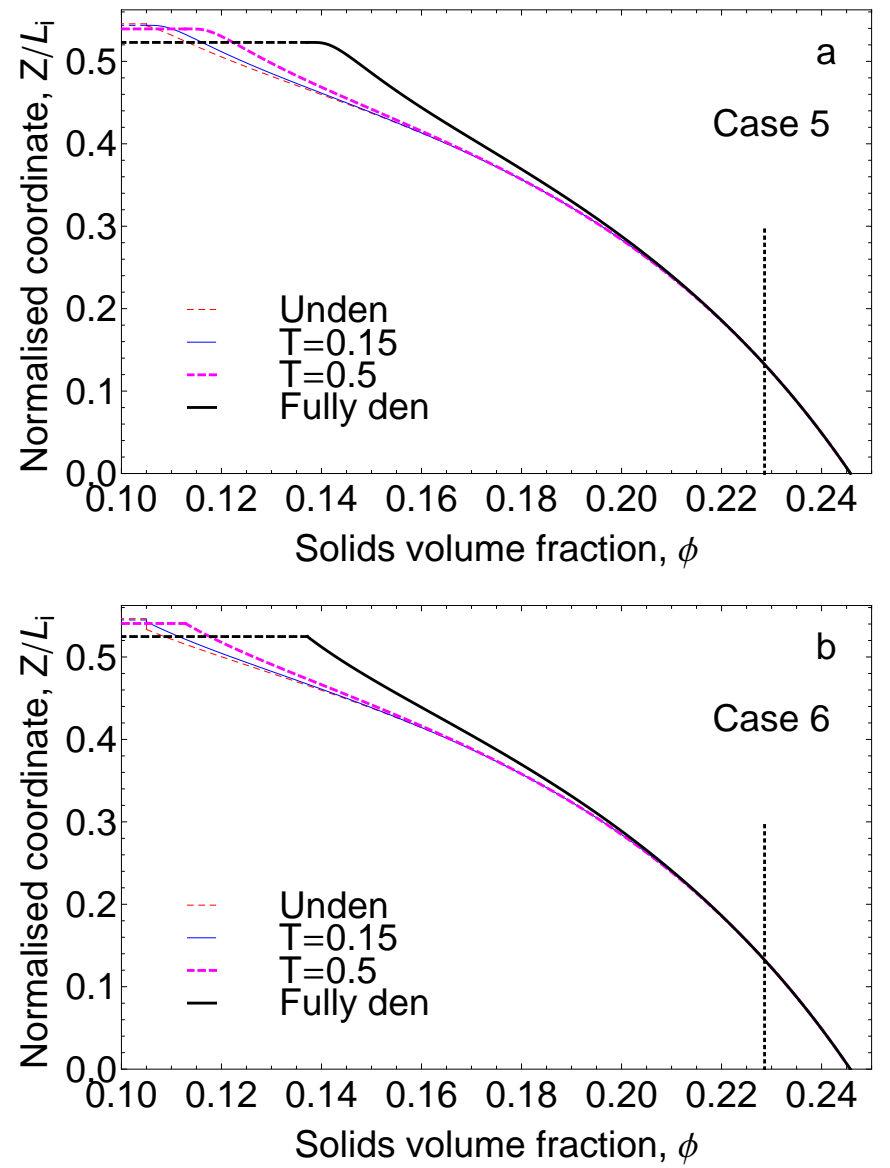

Figure 9: Predictions of the solids volume fraction profiles for Cases 5-6. Here the consolidated bed structures predicted for Case 5 (weak gel) and Case 6 (strong gel) are shown in the sub-plots labelled 'a' and 'b', respectively. The dotted vertical lines represent, in a fully densified state, the boundary between $\phi<\phi_{\text {agg }}$ (upper part of the bed) and $\phi>\phi_{\text {agg }}$ (lower part of the bed), densified suspension properties differing from undensified ones in the upper part only. At earlier times, with less densification, this boundary would be higher up in the bed. 

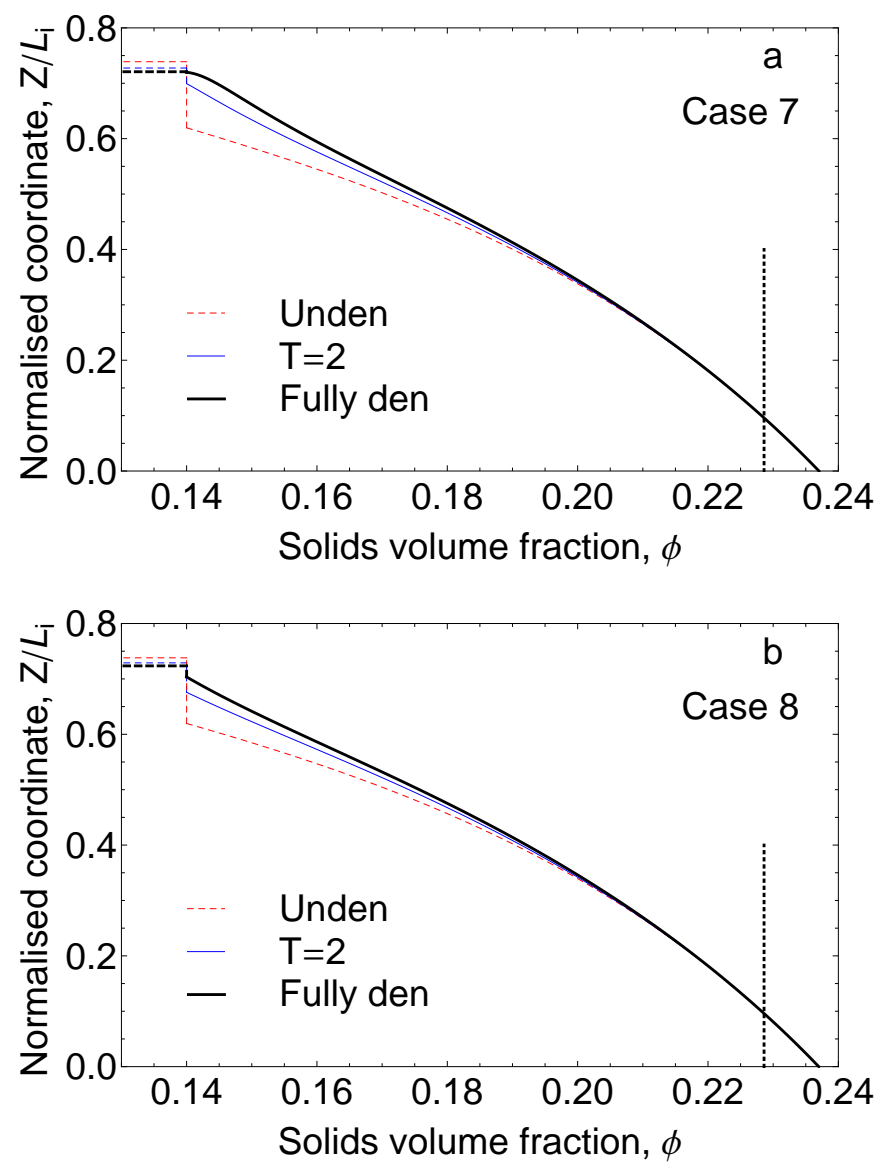

Figure 10: Determinations of the solids volume fraction profiles for Cases 7-8. The sub-plot labelled 'a' represents the consolidated bed structures predicted for Case 7 (weak gel) and the sub-plot labelled ' $b$ ' is for Case 8 (strong gel). The dotted vertical lines represent, in the case of the fully densified state, the boundary between the upper part of the consolidated bed (where $\phi<\phi_{\text {agg }}$ and densified sludge rheological properties are used) and the lower part of the consolidated bed (where $\phi>\phi_{a g g}$ and undensified sludge rheological properties are used). Again, at earlier times, with less densification, this boundary would be higher up in the bed. Recall that the initial feed solids volume fraction, $\phi_{f}$ equals 0.14 in Cases $7-$ 8. An unconsolidated zone (with uniform solids fraction $\phi_{f}$ ) can be seen above the top of the bed, and this shrinks but does not disappear altogether at long times. 